\title{
Supratubal recess and sinus tympani: CT analysis of middle ear hidden areas
}

\author{
Mohammad Waheed El-Anwar ${ }^{1 *}$ (D) Diaa Bakry Eldib², Ashraf Elmalt ${ }^{1}$ and Alaa Omar Khazbak ${ }^{1}$
}

\begin{abstract}
Background: High-resolution computed tomography (HRCT) magnifies the role of preoperative imaging for detailed middle ear anatomy particularly its hidden area. The purpose of the current study was to assess the sinus tympani (ST) and supratubal recess (STR) by HRCT, to create CT classification of the STR depth, and to study the relationship between ST types and the new STR grades.

Results: In HRCT of non-pathological temporal bones of 100 subjects (200 ears), measurements of the STR and ST were calculated, registered, and analyzed. The depth of the STR was classified into grade 1 with depth less than 3 $\mathrm{mm}$, grade 2 with depth ranged between 3 and $5 \mathrm{~mm}$, and grade 3 with depth more than $5 \mathrm{~mm}$. The mean STR length, width, and height were $4.17 \pm 0.86,3.55 \pm 0.65$, and $3.64 \pm 0.7 \mathrm{~mm}$, respectively, while the ST mean length and width of were $2.52 \pm 0.5$ and $1.82 \pm 0.78 \mathrm{~mm}$, respectively, without significant differences between either sexes or sided. The ST types were found to be type A in 56 ears (28\%), type B in 142 ears (71\%), and type $C$ in 2 ears (1\%). The STR grading was grade 1 in 12 ears (6\%), grade 2 in 160 ears (80\%), and grade 3 in 28 ears (14\%) without significant relationship between ST types and STR grading $(P=0.3)$.

Conclusion: The current study provided reliable and applicable methods of CT assessment of STR and ST that can help to predict the degree of surgical visibility of the ST and STR during ear surgery.
\end{abstract}

Keywords: Supratubal recess, Sinus tympani, Computed tomography, Anterior epitympanic space, Cholesteatoma

\section{Background}

In the last three decades, endoscope has been increasingly used in endonasal surgery that nowadays is simple and common [1]. Since the last decade, endoscope was used in otology firstly as a diagnostic tool then during middle ear surgery [2-7]. Endoscopy provided an important and sometimes unexpected detailed middle ear anatomy particularly for the hidden recesses such as the sinus tympani (ST) and the supratubal recess (STR). The STR is also known as the anterior epitympanic recess (AER) $[8,9]$.

Maximum exposure of the ST and STR in cholesteatoma surgery and complete removal of the disease is mandatory [10]. Endoscopic exploration of such hidden recesses (ST and STR) provides visualization and magnification that is almost impossible to achieve with microscopic traditional approaches without excess bone

\footnotetext{
* Correspondence: mwenteg1973@gmail.com; mwenteg@yahoo.com ${ }^{1}$ Otorhinolaryngology-Head and Neck Surgery Department, Faculty of Medicine, Zagazig University, Zagazig, Egypt

Full list of author information is available at the end of the article
}

drilling [11]. But endoscopic visualization of STR and ST could not be achieved in non-operated ears or after canal wall up mastoidectomy except during surgery. So, high-resolution computed tomography (HRCT) has a magnified role for the preoperative more detailed inner and middle ear anatomical information $[1,12]$ and it is the main guide for the surgeon to assess such hidden areas preoperatively and during surgery. However, in spite their crucial role in residual and recurrent disease, the ST and STR have received few and scattered attention in the radiological and otological literature and when they studied, many details are usually missed.

Recently, Marchioni et al. [13] classified the depth of the ST into three types (A, B, and C) (Fig. 1) based on the radiologic findings: type $\mathrm{A}$, small sinus tympani without medial or posterior extension in relation to the 3rd portion of facial nerve; type $B$, deep sinus tympani with medial extension but without posterior extension in relation to 3rd portion of the facial nerve; and type $\mathrm{C}$, large and deep sinus tympani with medial and posterior extension in relation to the 3rd portion of the facial 


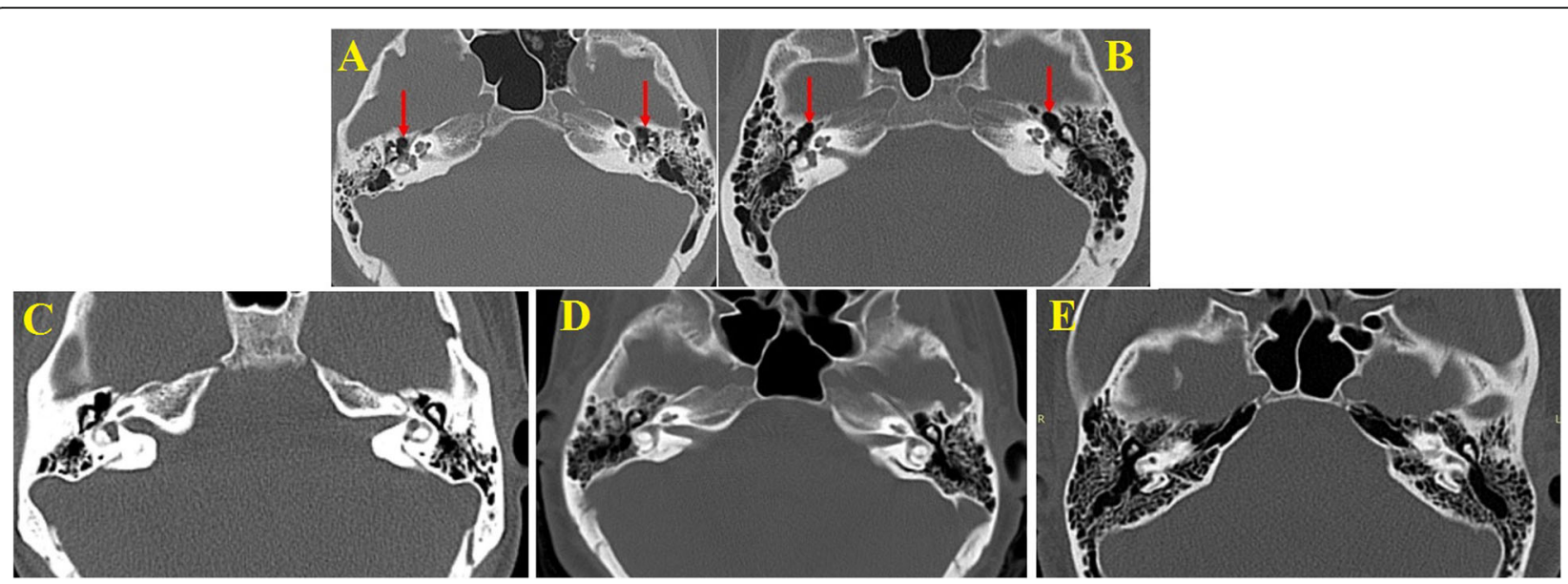

Fig. 1 a, b Supratubal recess (STR) (red arrows) in axial CT images and their relation to the ossicles and incudomalleal articulation. c The right and left STR formed of one cell. $\mathbf{d}$ The right STR formed of multi-cells while the left one formed of one cell. e Both the right and left STR) formed of multi-cells

nerve. Marchioni et al. [13] recommended further studies focused on ST types to prove their concept. Moreover, ST classification was not correlated with STR pneumatization and no classification of depth of STR had been developed yet.

The aim of the current study was to find an applicable way by HRCT imaging to assess the STR and ST in the way of proper preoperative review of the anatomy of middle ear. We aimed also to create an applicable CT classification of the depth of the STR and investigate the relation between ST types and our new STR grades.

\section{Methods}

\section{Subjects}

This study was conducted in the Otorhinolaryngology Department, in collaboration with the Radiodiagnosis Department. We retrospectively studied CT images of paranasal sinuses done for patients having symptoms of sinus-related complaints and who had no ear symptoms and no previous ear surgery. Randomly selected CT images of the non-pathologic temporal bones belonging to 100 subjects (200 ears) were included. The inclusion criterion of this study was the non-pathologic temporal bones. Informed written consent was signed by all subjects to share in the study after explanation of its purposes and the IRB approved the study.

Exclusion criteria included patients aged below 12 years, history of surgery or trauma in the skull base, congenital anomalies of the face, or paranasal sinus malignancies.

\section{Data acquisition and post-processing}

All examinations were done using multi-detector row CT a 64 slice CT scan (light speed volume VCT, GE medical system, Milwaukee, WI, USA) in helical mode. The raw data was exported by DICOM software, and the images were generated for the temporal region following temporal bone imaging protocol. Scan parameters include tube voltage of $120 \mathrm{kV}$ and a current of $180 \mathrm{~mA}$. Images were reconstructed using 0.5 - $\mathrm{mm}$-thick sections at 0.5 increments, using a $512 \times 512$ matrix of $0.43 \mathrm{~mm}$ pixel size and a field of view (FOV) of $22 \times 22 \mathrm{~cm}$. Images were displayed using a window center of $700 \mathrm{HU}$ and a window width of $4000 \mathrm{HU}$. CT images were analyzed and reviewed by all authors, and appropriate measurements were done by the radiologist author with more than 15 years of experience in CT imaging.

\section{Calculation (Figs. 1, 2, 3, and 4)}

The multiplanar reconstruction (MPR) technique was used to reconstruct the CT images and to produce coronal, sagittal, axial, and oblique axial images of the petrous temporal bones. All landmarks around the STR and ST were defined. To achieve uniformity throughout this craniometric study and to resolve the asymmetry associated with patient positioning, the Frankfort horizontal (Pfrk) plane was taken to be the standard horizontal plane, which is defined as the plane connecting the uppermost portions of the right and left external auditory canals (portions) and the inferior most portion of the infraorbital rim of the left eye. The sagittal plane (Psag) was vertical to the horizontal plane and was defined as the plane through the top of the crista galli and the middle point of the line from the central points of the right and left foramen spinosum. The coronal plane (Pcor) was defined as the plane through the line from the right and left foramen spinosum and was perpendicular to both horizontal and sagittal planes $[14,15]$.

\section{Measurements}

The multiplanar reconstruction (MPR) technique was used to reconstruct the $\mathrm{CT}$ images and to produce 


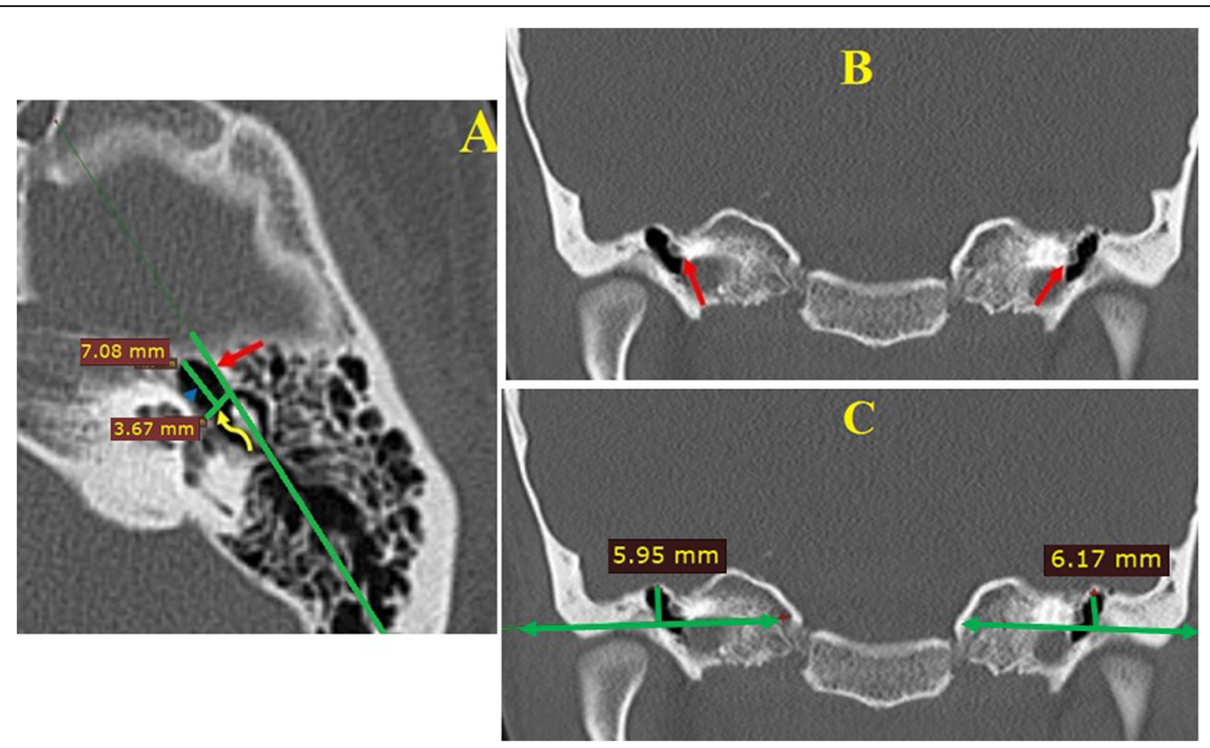

Fig. 2 Supratubal recess (STR) measurements. a Axial CT through the left STR, showing measurements of length (STR-L) and width (STR-W) of STR. STR-L: the deepest anterior to posterior diameter (arrowhead) parallel to incudomalleal axis (red arrow). STR-W: distance between medial and lateral borders of cog (curved yellow arrow). b, c Measurement of STR height (STR-H) in coronal CT, from tegmen tympani to the cochleariform process (red arrows), adjacent to the tensor tympani muscle. The cochleariform process separates the tensor tympani (hypoattenuating focus superolateral to the cochleariform process) from the bony Eustachian tube (inferomedially)

coronal, sagittal, and axial images of the petrous temporal bones to identify the STR (Figs. 1, 2, and 3).

The width of the STR (STR-W) was represented by the distance between the most lateral and inferior point to the most medial and inferior point of the cog in the axial plane. The length of the STR (STR-L) was represented by a line parallel to the axis of incudomalleolar articulation from the most anterior point of the STR to the line passing through the cog in axial plane (Figs. 1 and 2).

The height of the STR (STR-H) was represented by the superior-to-inferior (SI) distance from the tegmen tympani to the cochleariform process and the most superior point of the tympanic orifice of the ET, on the coronal plane [12, 16] (Fig. 2).
We classified the depth (antero-posterior) of the STR into three grades based on the radiologic findings; grade 1 STR-L where STR depth less than $3 \mathrm{~mm}$, grade 2 with STR depth ranged between 3 and $5 \mathrm{~mm}$, and grade 3 with STR depth more than $5 \mathrm{~mm}$ (Fig. 3).

The sinus tympani (ST) was identified in axial CT images with selection of the best image showing ST, promontory, and 3rd portion of the facial nerve canal. Types of ST (A, B, and C) identified and registered according to Marchioni et al. [13] classification.

The ST length (ST-L) was represented by the anteroposterior diameter of ST, from a tangential line passing through the anterior aspect of the facial nerve canal and the promontory to the deepest point of ST. The ST
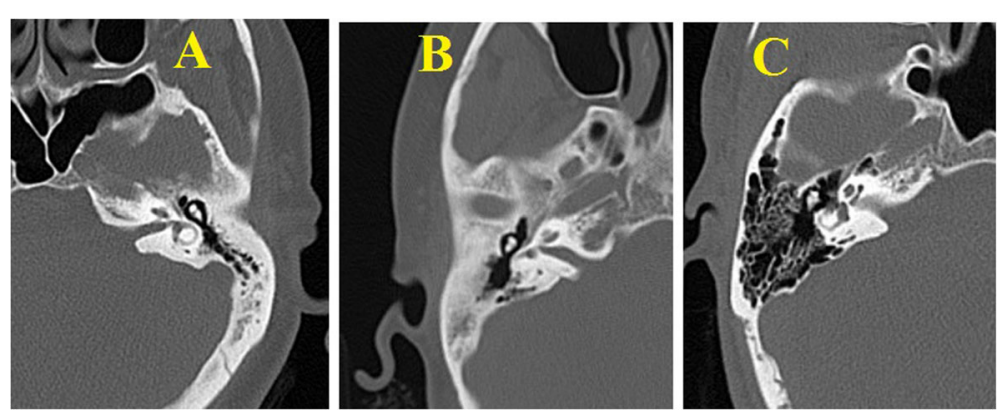

Fig. 3 Grades of the depth of STR. Axial CT images through the STR, showing the three grades of the depth of STR. a Grade 1: shallow STR. b Grade 2: medium-sized STR. c Grade 3: deep STR 


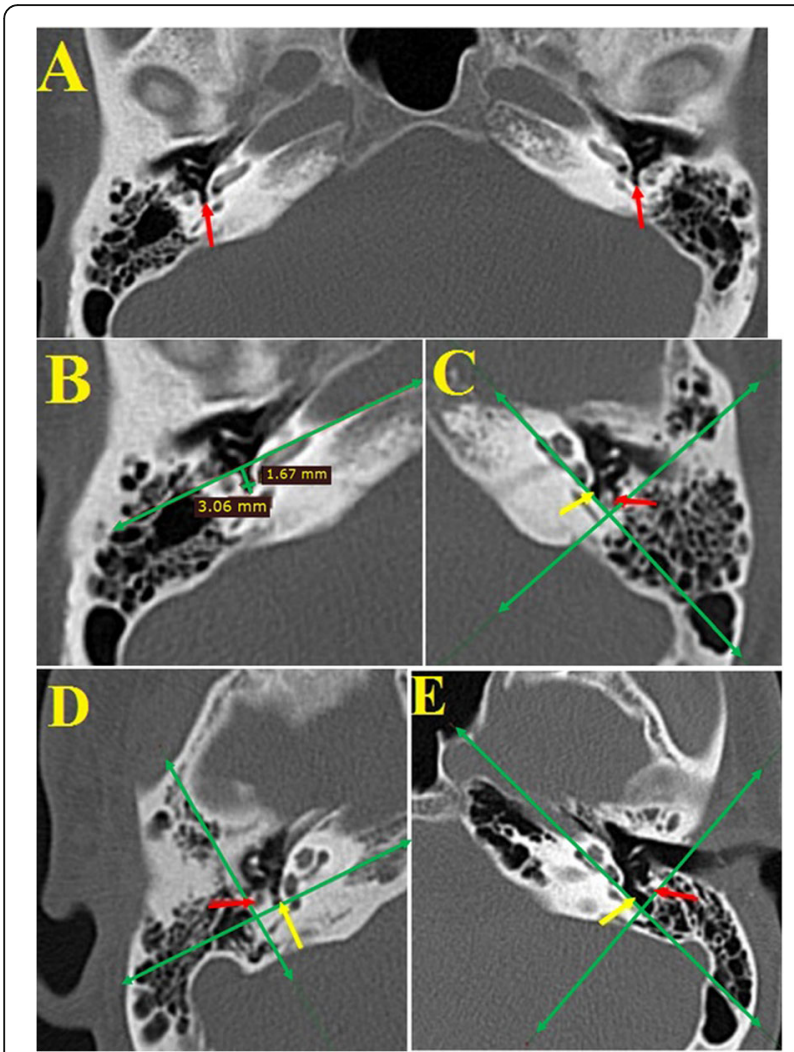

Fig. 4 Sinus tympani (ST) (STR) measurements and types. a Axial $\mathrm{CT}$ images showing the right and left Sinus tympani (ST) (red arrows). b Measurement of right ST length and width (ST-L \& ST-W) in axial CT image. c-e Different types of ST; yellow arrow points to ST and red arrow points to the facial nerve. The green lines passing through the floor of the ST and the outer wall of the facial nerve canal demonstrate the relation between ST and facial nerve. c Type A ST: small sinus tympani without medial or posterior extension in relation to the facial nerve. $\mathbf{d}$ Type B ST: deep sinus tympani with medial extension but without posterior extension in relation to the facial nerve. e Type C ST: large and deep sinus tympani with medial and posterior extension in relation to the facial nerve

width (ST-W) was represented by the widest transverse diameter of ST.

\section{Statistics}

All data are shown as means and standard deviation (SD). All statistical data were analyzed with the SPSS 25.0 (SPSS, Inc., Chicago, IL).

Results were compared statistically using the SPSS program version 25.0 (Chicago, Illinois, USA). When $P$ value was less than 0.05 , it was considered statistically significant.

\section{Results}

Two hundred ears of 100 subjects were included in the current study: 46 (46\%) females and 54 (54\%) males.
Their age ranged from 12 to 76 years; mean age was $38 \pm 13.29$ years (Table 1 ).

The ST types were found to be type A in 56 ears (28\%), type B in 142 ears (71\%), and type C in 2 ears (1\%) (Fig. 4).

The STR is formed of solitary cell in 159 ears $(79.5 \%)$ and multiple cells (multi-cells) in 41 ears (20.5\%) (Fig. 1).

The mean length (anterior-posterior depth) of the STR (STR-L) was $4.17 \pm 0.86 \mathrm{~mm}$ with a range of 2.36 to 7.85 $\mathrm{mm}(4.22 \pm 0.91 \mathrm{~mm}$ with a range of 2.36 to $7.44 \mathrm{~mm}$ at the right ears and $4.13 \pm 0.81 \mathrm{~mm}$ with a range of 2.73 to $7.85 \mathrm{~mm}$ at the left ears), while the mean length in male was $4.28 \pm 0.84 \mathrm{~mm}$ (range 2.73-6.97) and in female was $4.05 \pm 0.88 \mathrm{~mm}$ (range 2.36-7.85). No significant difference was observed in the length of the STR between the right and left ears $(P<0.4609)$ or between male and female subjects $(P<0.0605)$ (Table 1$)$.

The STR grading of its anterior-posterior depth was grade $1(<3 \mathrm{~mm})$ in 12 ears $(6 \%)$, grade $2(3-5 \mathrm{~mm})$ in 160 ears $(80 \%)$, and grade $3(>5 \mathrm{~mm})$ in 28 ears $(14 \%)$ (Table 2).

The mean width of the STR (STR-W) was $3.55 \pm 0.65$ $\mathrm{mm}$ with a range of 1.04 to $5.57 \mathrm{~mm}$. The mean width was $3.52 \pm 0.62 \mathrm{~mm}$ with a range of 2.37 to $5.48 \mathrm{~mm}$ at the right ears and $3.59 \pm 0.68 \mathrm{~mm}$ with a range of 1.04 to $5.57 \mathrm{~mm}$ at the left ears, while the mean width in male was $3.58 \pm 0.7 \mathrm{~mm}$ with a range of 1.04 to $5.57 \mathrm{~mm}$ and in female was $3.52 \pm 0.6$ with a range of 2.04 to $5.48 \mathrm{~mm}$. No significant difference was observed in the width of the supratubal recess (STR-W) between the right and left ears $(P<0.4477)$ or between male and female subjects $(P<0.5172)$ (Table 1$)$.

The mean height (superior-inferior depth) of the STR (STR-H) was $3.64 \pm 0.7 \mathrm{~mm}$ with a range of 2.3 to 5.88 $\mathrm{mm}(3.6 \pm 0.68 \mathrm{~mm}$ with a range of 2.37 to $5.88 \mathrm{~mm}$ at the right ears and $3.68 \pm 0.7 \mathrm{~mm}$ with a range of 2.33 to $5.74 \mathrm{~mm}$ at the left ears). While the mean height in male was $3.66 \pm 0.75 \mathrm{~mm}$ with a range of 2.33 to $5.88 \mathrm{~mm}$ and in female was $3.61 \pm 0.62$ with a range of 2.3 to $5.45 \mathrm{~mm}$. No significant difference was observed in the height (superior-inferior depth) of the STR (STR-H) between the right and left ears $(P<0.474)$ or between male and female subjects $(P<0.6118)$ (Table 1$)$.

The mean length of the ST (ST-L) was $2.52 \pm 0.5 \mathrm{~mm}$ with a range of 1.36 to $3.97 \mathrm{~mm}(2.51 \pm 0.51 \mathrm{~mm}$ with a range of 1.36 to $3.63 \mathrm{~mm}$ at the right ears and $2.52 \pm 0.5$ $\mathrm{mm}$ with a range of 1.39 to $3.97 \mathrm{~mm}$ at the left ears), while the mean length in male was $2.47 \pm 0.5 \mathrm{~mm}$ with a range of 1.39 to $3.63 \mathrm{~mm}$ and in female was $2.56 \pm 0.5$ with a range of 1.36 to $3.97 \mathrm{~mm}$. No significant difference was observed in the length of the sinus tympani (ST-L) between the right and left ears $(P<0.8888)$ or between male and female subjects $(P<0.206)$ (Table 1$)$. 
Table 1 Analysis of the STR and ST measurements using mean \pm SD and range (in millimeter)

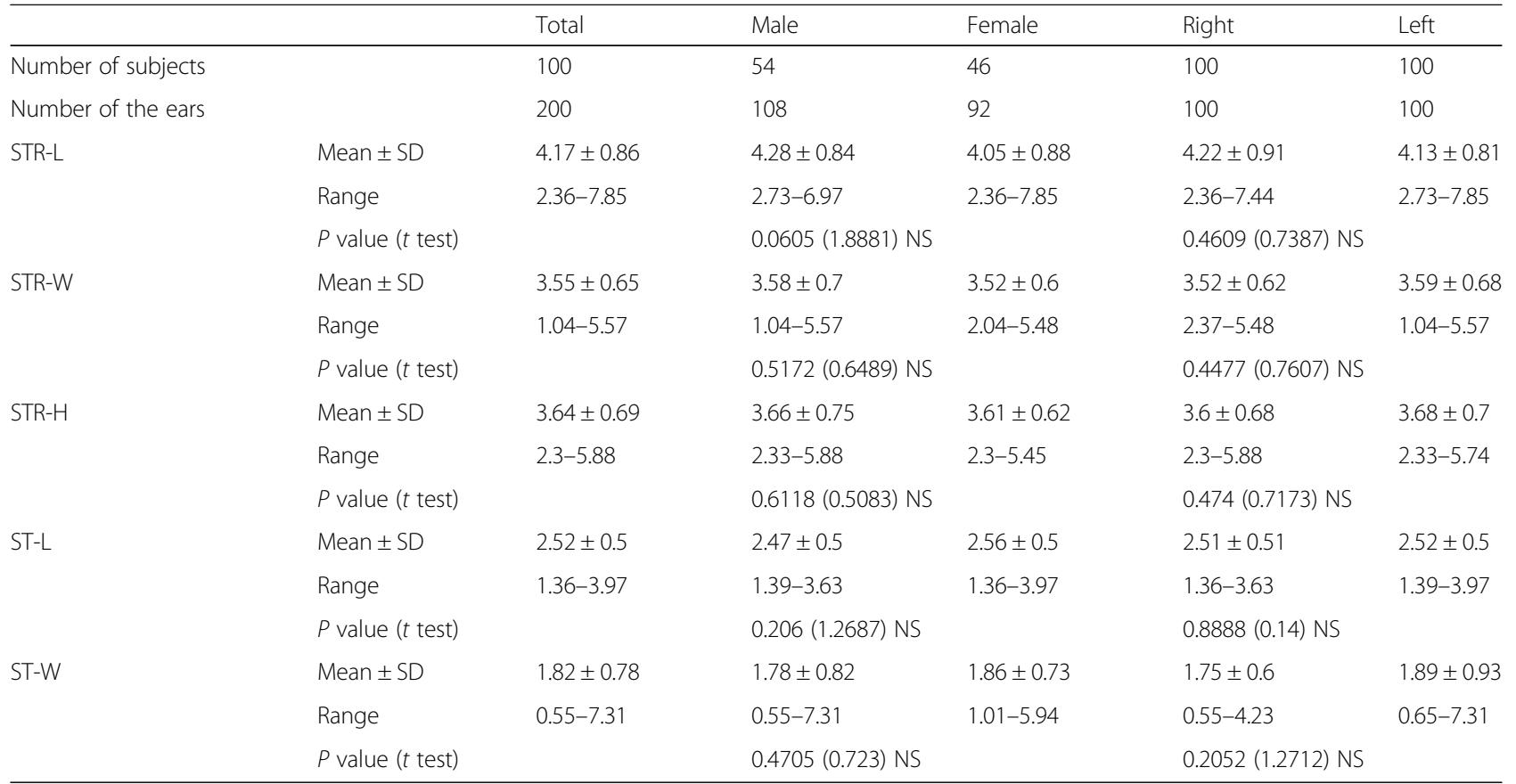

STR supratubal recess, ST sinus tympani, STR-L STR length, STR-W STR width, STR-H STR height, ST-L ST length, ST-W ST width, SD Standard deviation, NS Non-significant

The mean width of the ST (ST-W) was $1.82 \pm 0.78 \mathrm{~mm}$ with a range of 0.55 to $7.31 \mathrm{~mm}(1.75 \pm 0.6 \mathrm{~mm}$ with a range of 0.55 to $4.23 \mathrm{~mm}$ at the right ears and $1.89 \pm 0.93$ $\mathrm{mm}$ with a range of 0.65 to $7.31 \mathrm{~mm}$ at the left ears), while the mean width in male was $1.78 \pm 0.82 \mathrm{~mm}$ with a range of 0.55 to $7.31 \mathrm{~mm}$ and in female was $1.86 \pm 0.73$ with a range of 1.01 to $5.94 \mathrm{~mm}$. No significant difference was observed in the width of the sinus tympani (ST-W) between the right and left ears $(P<0.2052)$ or between male and female subjects $(P<0.4705)$ (Table 1$)$.

No significant relationship was detected between ST types and STR grading $\left(P=0.3, X^{2}=4.874\right)$ (Table 3$)$.

\section{Discussion}

Our study highlighted two major anatomic landmarks and sites in the middle ear (STR and ST); one should be familiar with these landmarks to be able to describe normal and pathologic middle ear findings and provide an accurate CT interpretation and preoperative surgical assessment. HRCT is a major tool for imaging evaluation of the middle ear. HRCT offers excellent delineation of soft tissue abnormalities against a background of air

Table 2 STR length (anterior-posterior depth) grading

\begin{tabular}{llll}
\hline & Grade 1 & Grade 2 & Grade 3 \\
\cline { 2 - 3 } & $<3 \mathrm{~mm}$ & $3-5 \mathrm{~mm}$ & $>5 \mathrm{~mm}$ \\
Measurement in $\mathrm{mm}$ & 12 & 160 & 28 \\
Percent & $6 \%$ & $80 \%$ & $14 \%$ \\
\hline
\end{tabular}

(middle ear cavity, EAC, mastoid air cells) and allows excellent preoperative assessment of bone changes and/or involvement.

The STR is a common site of recurrent cholesteatoma, in both congenital and acquired cholesteatomas; however, it was not clearly recognized why cholesteatoma formed so easily in the STR [9]. The prevalence of residual cholesteatoma is high following mastoidectomy. Even though, otoendoscopy is helpful to reduce retrotympanic residual cholesteatoma, anterior attic (STR) residual still represents a problem particularly with the canal wall up technique [17]. Thus, the surgeons directed their attention to the ST and STR due to their frequent involvement by cholesteatoma and their relations to important structures. But, the STR and ST have received scanty attention and in the radiological literature [12, 17-19] without detailed description. We tried in our current study to use HRCT to assess both STR

Table 3 Supratubal recess (STR) grading in relation to sinus tympani (ST) types

\begin{tabular}{llllll}
\hline $\begin{array}{l}\text { ST type } \\
\text { STR grade }\end{array}$ & Type A & Type B & Type C & Total & $P$ value \\
\hline Grade 1 & $4(33 \%)$ & $8(67 \%)$ & $0(0 \%)$ & $12(6 \%)$ & 0.3 NS \\
Grade 2 & $41(25.6 \%)$ & $118(73.8 \%)$ & $1(0.6 \%)$ & $160(80 \%)$ & $X^{2}=4.874$ \\
Grade 3 & $11(39 \%)$ & $16(57 \%)$ & $1(4 \%)$ & $28(14 \%)$ & \\
Total & $56(28 \%)$ & $142(71 \%)$ & $2(1 \%)$ & $200(100 \%)$ & \\
\hline
\end{tabular}

NS non-significant, $X^{2}$ chi-square 
and ST in details defining their measurements in all directions and provide a new classification of the STR.

HRCT of the temporal bone is therefore a useful guide to the surgeon because it provides information regarding the extent of disease as well as possible anatomic variations and potential complications that may be avoided during surgery [20]. We used 64 HRCT scanner that is sufficient to improve the image quality and better delineation of middle ear structures [21, 22].

Effective doses in sinus CT are 0.5-0.9 mSv and in ear CT are $0.3-0.6 \mathrm{mSv}$ [23]. Because CT examinations of the paranasal sinus and ear presented the highest dose to eye lens of $35.3 \mathrm{mGy}$ [24], lead lined eye shield was used in the current work to minimize exposure of the eye and lens to radiation.

The ST is a posterior outpouching of the retrotympanum delimited by the mastoid part of the facial nerve and pyramidal eminence laterally and vestibule medially [19, 24] (Fig. 4).

During cholesteatoma surgery, the cholesteatoma may be located and missed in the depth of the ST that represents an important surgical challenge due to the conformation and position of the ST [25]. Marchioni et al. [19] stated that when cholesteatoma involves the ST, there are two clinically important risks: first is the potential risk of residual disease due to incomplete removal of the cholesteatoma, and second is the increased risk for ossicular discontinuity and hearing loss due to cholesteatoma within the ST. Therefore, the CT-derived morphology of the ST could help the surgeon in the preoperative assessment [13].

In our current retrospective study on 200 ears CT, we confirm the data published by Marchioni et al. [13] who reviewed HRCT scan of the temporal bones. We were able to confirm the presence of their described three ST variations and codify their incidence: $28 \%$ for type A, $71 \%$ for type B, and $1 \%$ for type C. So, based on our data, ST type B is the most common morphological ST conformation, and ST Type A is less common that is similar to previous findings $[13,19]$. In addition, we defined the exact measurements of the ST.

On the other hand, STR is bounded anteriorly by the root of zygomatic arch, medially by the anterior part of the tympanic facial nerve and geniculate ganglion, posteriorly by the $\operatorname{cog}$, laterally by the scutum, and superiorly by the tegmen tympani (separates it from the dura mater) [12].

The STR could be involved by different pathologic processes. In both congenital and acquired cholesteatomas, the STR has been shown to be the common site of recurrence $[12,18]$. Familiarity with the $\mathrm{CT}$ appearance of this space facilitates recognition of its early pathologic changes or its involvement in extensive lesions.

Our study shows that the STR is an anatomic structure that is consistently identified on HRCT scans. Thus, preoperative CT assessment will give orientation for STR configuration.

We reported that STR is commonly formed of solitary cell $(79.5 \%)$, while in $20.5 \%$ of the ears, it is formed of multiple cells. These results are in agreement with the results of the recent study of Hong et al. [9]. STR depth in the current study was $4.17 \pm 0.86 \mathrm{~mm}$ with a range of 2.36 to 7.85 that is near to mean detected by Marchioni et al. [12] (5.1 $\pm 1.46 \mathrm{~mm})$.

We introduced a new grading system for classification of the depth (anterior-posterior depth) of the STR into three grades based on the HRCT findings: grade 1 (depth $<3 \mathrm{~mm}$ ), grade 2 (depth $3-5 \mathrm{~mm}$ ), and grade 3 (depth $>5 \mathrm{~mm}$ ). We found that grade 2 STR was the most common (80\%) followed by the deep STR (grade 3, 14\%) while the shallow STR (grade 1) was the lease common (6\%).

We found that there was no significant relationship between ST types and STR grading, so the depth of each of them is not associated with or expect depth of the other recess and the STR and ST should be assessed radiologically as a separate entity.

In deep STR (types 2 and 3), surgeons need to be ready to use of angled $\left(45^{\circ}\right)$ endoscope and drill the anterior portion of the scutum mostly.

These newly suggested preoperative CT measurements and new STR grades are applicable, measurable in children and adult, and reliable. Therefore, they can be used as good predictors for the STR visibility during cholestetoma surgery and predict the need for the incus and malleus head removal and or otoendoscopy use to access the recess with different measurement. In addition, these will help to increase attention of the radiologists and the otosurgeons to the CT evaluation of these important regions.

It seems intuitive that the deeper the ST and the larger STR, the higher the risk of residual disease. So, second look surgery could be expected more in type C ST and grade 3 STR. Adding of the ST types and STR grading to the preoperative CT check list is recommended. Further studies focused on that topic in cholesteatoma patients are required.

Our study has some limitations; the study was conducted on non-pathological temporal bones, and further studies discussing changes in ST and STR measurements in cases of cholesteatoma are required. Also, our study did not discuss the age-related changes in ST and STR parameters due to lack of a considerable number of subjects in childhood age.

\section{Conclusion}

The described measurements and grading of STR and ST allow otosurgeons and radiologist to assess such crucial and potentially hidden areas. The degree of surgical visibility of the ST and STR during middle ear surgery can depend on these measurements and classification. 


\section{Abbreviations}

ET: Eustachian tube; HRCT: High-resolution computed tomography; MPR: Multiplanar reconstruction; SD: Standard deviation; ST: Sinus tympani; STR: Supratubal recess; STR-H: Height of the supratubal recess; STR-L: Length of the supratubal recess; STR-W: Width of the supratubal recess

\section{Acknowledgements}

The authors thank all the study participants for their patience and support.

\section{Authors' contributions}

MWE suggested and developed the research idea; followed up the work progress; reviewed literature; defined the study protocol, interpretation, and analysis of collected data; wrote the manuscript; assisted in preparing the figures; performed the statistical analysis; followed up the work progress; and approved the submitted version. DBE performed the data acquisition and post-processing; performed CT assessment and measurements; kept the record of patients' information; tabulated data, interpretation, and analysis of collected data; assisted in writing methods and revising the written manuscript; prepared the figures, and approved the submitted version. AOK made initial arrangements and coordination with the Radiodiagnosis Department, reviewed the written manuscript, revised the manuscript critically for important intellectual content, and approved the submitted version. AE reviewed the written manuscript, revised the manuscript critically for important intellectual content, and approved the submitted version. All authors have read and approved the manuscript.

\section{Funding}

The authors declare no financial support or interest to this study.

\section{Availability of data and materials}

The datasets used and/or analyzed during the current study are available from the corresponding author on reasonable request.

\section{Ethics approval and consent to participate}

Informed written consent was signed by all subjects to share in the study after explanation of its purposes. Zagazig University IRB approved the study (2016).

\section{Consent for publication}

All patients included in this research gave written informed consent to publish the data contained within this study. If the patient was less than 16 years old, deceased, or unconscious when consent for publication was requested, written informed consent for the publication of this data was given by their parent or legal guardian.

\section{Competing interests}

The authors declare that they have no competing interests.

\section{Author details}

Otorhinolaryngology-Head and Neck Surgery Department, Faculty of Medicine, Zagazig University, Zagazig, Egypt. ${ }^{2}$ Radiodiagnosis Department, Faculty of Medicine, Benha University, Benha, Egypt.

Received: 6 June 2019 Accepted: 23 September 2019

Published online: 18 November 2019

\section{References}

1. Prevedello DM, Doglietto F, Jane JA Jr et al (2007) History of endoscopic skull base surgery: its evolution and current reality. J Neurosurg 107:206-213

2. Baki FA, El Dine MB, El Said L et al (2002) Sinus tympani endoscopic anatomy. Otolaryngol Head Neck Surg 127:158-162

3. Thomassin JM, Korchia D, Doris JM (1993) Endoscopic guided otosurgery in the prevention of residual cholesteatomas. Laryngoscope 103:939-943

4. Badr-El-Dine M (2002) Value of ear endoscopy in cholesteatoma surgery. Otol Neurotol 23:631-635

5. Tarabichi M (1997) Endoscopic management of acquired cholesteatoma. Am J Otol 18:544-549

6. Tarabichi M (2004) Endoscopic management of limited attic cholesteatoma. Laryngoscope 114:1157-1162

7. Elfeky AEM, Khazbzk AO, Nasr WF. et al. (2019) Outcomes of Using Otoendoscopy During Surgery for Cholesteatoma. Indian J Otolaryngol
Head Neck Surg 71(Suppl 2):1036. https://doi.org/10.1007/s12070-0171084-7.

8. Cohen D, Blinder G, Perez R, Raveh D (2005) Standardized computed tomographic imaging and dimensions of the round-window niche. Int Tinnitus J 11(2):158-162

9. Hong J, Chen K, Lyu H, Yin D, Yang L, Zhang T, Dai P (2018) Age-related changes in the morphological relationship between the supratubal recess and the Eustachian tube. Auris Nasus Larynx 45:88-95

10. Marchioni D, Alicandri-Ciufelli M, Piccinini A, Genovese E, Presutti L (2010) Inferior retrotympanum anatomy revisited: an endoscopic anatomic study. Laryngoscope 120(9):1880-1886

11. Marchioni MG, Presutti $L$ (2011) Endoscopic anatomy of the middle ear. Indian J Otolaryngol Head Neck Surg 63(2):101-113

12. Marchioni D, Mattioli F, Cobelli M, Todeschini A, Alicandri-Ciufelli M, Presutti $L$ (2009) CT morphological evaluation of anterior epitympanic recess in patients with attic cholesteatoma. Eur Arch Otorhinolaryngol 266:1183-1189

13. Marchioni VS, Mattioli F, Alicandri-Ciufelli M, Presutti L (2015) Radiological assessment of the sinus tympani: temporal bone HRCT analyses and surgically related findings. Surg Radiol Anat 37:385-392

14. Lyu HY, Chen KG, Yin DM, Hong J, Yang L, Zhang TY et al (2016) The agerelated orientational changes of human semicircular canals. Clin Exp Otorhinolaryngol 9:109-115

15. Chen K, Yin DM, Lyu HY, Yang L, Zhang TY, Dai PD (2016) Anatomic measurements of the posterior tympanum related to the round window vibroplasty in congenital aural atresia and stenosis patients. Acta Otolaryngol 136:1-5

16. Petrus LV, Lo WW (1997) The anterior epitympanic recess: CT anatomy and pathology. Am J Neuroradiol 18:1109-1114

17. Gaillardin L, Lescannea E, Morinière S, Cottierb JP, Robiera A (2012) Residual cholesteatoma: prevalence and location. Follow-up strategy in adults. Eur Ann Otorhinolaryngol Head Neck Dis 129:136-140

18. Rosito LS, Netto LF, Teixeira AR, da Costa SS (2016) Classification of cholesteatoma according to growth patterns. JAMA Otolaryngol Head Neck Surg 142:168-172

19. Marchioni D, Mattioli F, Alicandri-Ciufelli M, Presutti L (2009) Transcanal endoscopic approach to the sinus timpani: a clinical report. Otol Neurotol 30(6):758-765

20. Abdel Razek A, Huang BY (2012) Lesions of the petrous apex: classification and findings at CT and MR imaging. Radiographics 32:151-173

21. Razek AA, Ezzat A, Azmy E, Tharwat N (2013) Role of whole-body 64-slice multidetector computed tomography in treatment planning for multiple myeloma. Radiol Med 118:799-805

22. Razek AA, Ghonim MR, Ashraf B (2015) Computed tomography staging of middle ear cholesteatoma. Pol J Radiol 80:328-333

23. Valentin J (2007) The 2007 recommendations of the international commission on radiological protection. ICRP publication 103. Ann ICRP 37(2):1-332

24. Yamauchi-Kawaura C, Fujii K, Aoyama T, Yamauchi M, Koyama S (2009) Evaluation of radiation doses from MDCT-imaging in otolaryngology. Radiat Prot Dosim 136(1):38-44

25. Pickett BP, Cail WS, Lambert PR (1995) Sinus tympani: anatomic considerations, computed tomography, and a discussion of the retrofacial approach for removal of disease. Am J Otol 16:741-750

\section{Publisher's Note}

Springer Nature remains neutral with regard to jurisdictional claims in published maps and institutional affiliations. 